\title{
An Analysis of Models Describing Predator-Prey Interaction
}

RAYMOND P. CANALE, Department of Civil Engineering, The University of Michigan, Ann Arbor, Michigan 48104

\begin{abstract}
Summary
Mathematical models of the interaction between predator and host populations have been expressed as systems of nonlinear ordinary differential equations. Solutions of such systems may be periodic or aperiodic. Periodic oscillatory solutions may depend on the initial conditions of the system or may be limit cycles. Aperiodic solutions can, but do not necessarily, exhibit oscillatory behavior. Therefore, it is important to characterize predatory-prey models on the basis of the possible types of solutions they may possess. This characterization can be accomplished using some well-known methods of nonlinear analysis. Examination of the system singular points and inspection of phase plane portraits have proved to be useful techniques for evaluating the effect of various modifications of early predator-prey models. Of particular interest is the existence of limit cycle oscillations in a model in which predator growth rate is a function of the concentration of prey.
\end{abstract}

\section{INTRODUCTION}

Lotka ${ }^{1}$ and Volterra ${ }^{2}$ utilized nonlinear differential equations to assist their study of predator-prey relationships. The results of their investigations for batch cultures are well known and are given by eq. (1) and eq. (2):

$$
\begin{aligned}
& \frac{d H}{d t}=\mu H-k_{1} H P \\
& \frac{d P}{d t}=k_{2} H P-\delta P
\end{aligned}
$$

where $t=$ time, $H=$ concentration of hosts (prey), $P=$ concentration of predators, $k_{1}=$ constant, $k_{2}=$ constant, $\mu=$ specific growth rate of the hosts, and $\delta=$ specific death rate of the predators.

(C) 1970 by John Wiley \& Sons, Inc. 
Bungay and Bungay ${ }^{3}$ have written similar equations for a completely mixed culture with a continuous input of sterile medium. These equations are:

$$
\begin{gathered}
\frac{d H}{d t}=\mu H-D H-k_{1} H P \\
\frac{d P}{d t}=k_{2} H P-D P
\end{gathered}
$$

where $D$ is the dilution rate. Equations (3) and (4) have incorporated the assumption that the overflow of predator and prey, $D P$ and $D H$, is much larger than the decrease in population due to death. Such an assumption seems justified except for very low dilution rates. Second order kinetic expressions have been used to describe both the predator growth and the host consumption.

A critical examination of eqs. (1) and (2) and eqs. (3) and (4) reveals some obvious difficulties. Equation (1) and eq. (3) indicate that in the absence of predator populations the host will increase exponentially with time. This unrealistic behavior can be altered by assuming that the specific growth rate of the host is a function of some nutrient in the environment,

$$
\mu=\mu(s)
$$

where $s$ is the concentration of a limiting nutrient. A functional relationship based on second order kinetics can be proposed for the growth of the host. This is similar to the original Lotka-Volterra assumptions and results in eq. (6) for $\mu(s)$ :

$$
\mu=\hat{\mu} \frac{s}{\hat{s}}
$$

where $\hat{\mu}$ and $\hat{s}$ are any reference values for $\mu$ and $s$ as illustrated in Figure 1. Combining eqs. (3), (4), and (6), and writing a material balance equation for $s$ results in the following model:

$$
\begin{gathered}
\frac{d s}{d t}=D\left(s_{I}-s\right)-\frac{\widehat{\mu}}{\mathrm{Y}} \frac{s}{\hat{s}} H \\
\frac{d H}{d t}=\hat{\mu} \frac{s}{\hat{s}} H-D H-\frac{\hat{\eta}}{W} \frac{H}{\hat{H}} P
\end{gathered}
$$




$$
\frac{d P}{d t}=\hat{\eta} \frac{H}{\hat{H}} P-D P
$$

where $s_{I}=$ concentration of $s$ in the inflowing medium, $Y=$ host yield constant, host produced/substrate consumed, and $W=$ predator yield constant, predator produced/host consumed and where the following definitions have been introduced

$$
\begin{aligned}
& k_{2} H=\eta(H)=\hat{\eta} \frac{H}{\hat{H}} \\
& k_{1} H=\frac{\eta(H)}{W}=\frac{\hat{\eta}}{W} \frac{H}{\hat{H}}
\end{aligned}
$$

The model given by eqs. (7), (8), and (9) assumes that the host and predator yields are constant. No claim can be made that such an assumption is exactly true in any specific food chain over long periods of time. However, experimental studies by Monod, ${ }^{4}$ Herbert et at., ${ }^{5}$ Schultze ${ }^{6}$ Proper and Garver ${ }^{7}$ and Curds and Cockburn ${ }^{8}$ suggest that the assumptions are approximately true for the food chain:

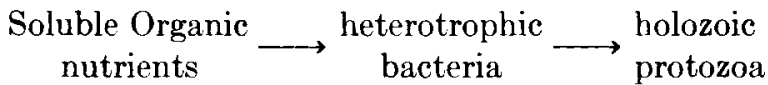

The Lotka-Volterra model and the model proposed by eqs. (7), (8), and (9) do not indicate the existence of an upper limit on the host or

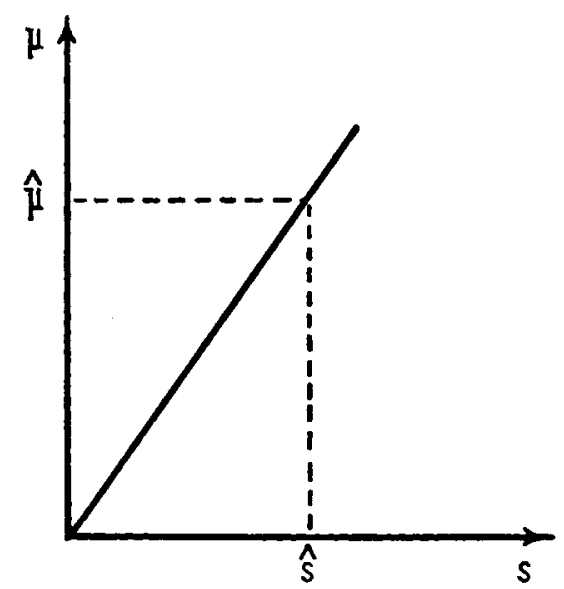

Fig. 1. Linear variation of $\mu$ and $S$. 
predator specific growth rate. Monod ${ }^{4}$ introduced the following function to account for saturation of bacterial specific growth rates,

$$
\mu=\mu_{\max } \frac{s}{K+s}
$$

where $K$ is a constant. Proper and Garver ${ }^{7}$ have used a similar expression to describe the specific growth rate of a protozoan population as a function of its food supply,

$$
\eta=\eta_{\max } \frac{H}{L+H}
$$

By introducing eqs. (10) and (11) into eqs. (7), (\$), and (9) a more refined model is obtained as given by eqs. (12), (13), and (14):

$$
\begin{gathered}
\frac{d s}{d t}=D\left(s_{I}-s\right)-\frac{\mu_{\max }}{Y} \frac{s H}{K+s} \\
\frac{d H}{d t}=\mu_{\max } \frac{s H}{K+s}-D H-\frac{\eta_{\max }}{W} \frac{H P}{L+H} \\
\frac{d P}{d t}=\eta_{\max } \frac{H P}{L+H}-D P
\end{gathered}
$$

The model as given by eqs. (12), (13), and (14) has been described previously by Drake et al., ${ }^{9}$ Bungay and Bungay, ${ }^{3}$ and Canale ${ }^{10,11}$ for bacterium-protozoan systems.

It is important to be able to characterize the behavior of the solutions of the above models. For example, the solutions of the LotkaVolterra equations are a family of closed curves on the HP-plane. These closed curves represent a cyclic variation of both $H$ and $P$ with time. Nuch interest has been expressed by the scientific community regarding the existence or nonexistence of such cycles in nature. In 1934 Gause $^{12}$ published his famous experiments on predation in a protozoan system. Slobodkin ${ }^{13}$ has discussed the reality of cycles in nature. A recent monograph by Salt ${ }^{14}$ has reviewed much of the writings on predation.

The Lotka-Volterra model is easy to solve for the relationships between $H$ and $P$. The solutions of the modified predator-prey models are much more difficult to obtain. Both modified forms are highly nonlinear and general solutions have not been found. The BIOTECHNOLOGY AND BIOENGINEERING, VOL. XII, ISSUE 3 
purpose of this paper is to use the methods of nonlinear mechanics to study the behavior of the solutions of the modified models. It is of interest to know whether periodic solutions are possible in the modified models. If periodic solutions exist, are they limit cycles or oscillations which depend on the system initial conditions? It is hoped that such a study will increase our insight into the basic biological mechanisms involved in predator-prey interactions.

\section{Phase Plane Analysis}

The behavior of nonlinear predator-prey models may be studied by a technique known as phase plane analysis. As an introduction to this method of analysis, consider a system of two first order differential equations in the general, unspecified variables $x$ and $y$ :

$$
\begin{aligned}
& \frac{d x}{d t}=\mathrm{F}(x, y) \\
& \frac{d v}{d t}=G(x, y)
\end{aligned}
$$

The goal will not be that of finding solutions of the equations, but rather that of determining the possible geometric configurations of the solution curves. The solution curves will be studied on the $x y$-plane with the independent variable $t$ treated as a parameter. A curve is thus traced which may have a variety of configurations as shown in Figure 2. The arrows show the direction in which the point $P(x, y)$ moves as $t$ varies from $-\infty$ to $+\infty$.

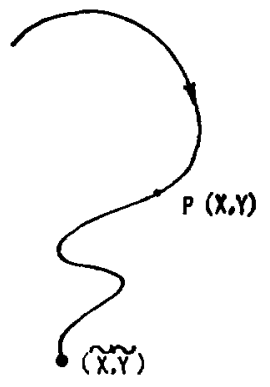

A STABLE MOTION

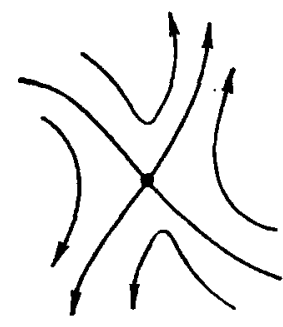

B UNSTABLE MOTION

Fig. 2. Phase plane configurations. 
A point $(\tilde{x}, \tilde{y})$ at which both $F(x, y)$ and $G(x, y)$ are zero is called an equilibrium point, or a singular point. A singular point is called isolated if a circle exists which has a center at $(\bar{x}, \tilde{y})$ and contains no other singular points. The singular points of a system are important since they denote stationary values of $x$ and $y$. If the system initial conditions happen to be at a singular point, then the solution of the system is given by the equations $x=\bar{x}, y=\tilde{y}$ for all $t$. In Figure $2 \mathrm{a}$, $P(x, y)$ approaches the singular point in the limit as $t$ approaches $+\infty$. The resulting motion converges toward the singular point and the singular point is called stable. If however, the arrows point outward and diverge from the singular point as $t$ approaches $+\infty$ the singular point and resulting motion are considered unstable. Figure $2 \mathrm{~b}$ illustrates such a case.

Consider a system represented by eqs. (15) with an isolated singular point $(\tilde{x}, \tilde{y})$ at the origin where $\bar{x}=0, \tilde{y}=0$. If the singular point is not located at the origin, a simple translation $u=x-\tilde{x}, v=$ $y-\tilde{y}$, can be imposed. To study the solutions near the singular point expand $F(x, y)$ and $G(x, y)$ in a power series:

$$
\begin{aligned}
& \frac{d x}{d t}=a_{1} x+b_{1} y+c_{1} x^{2}+d_{1} x y+\mathrm{e}_{1} y^{2}+\ldots \\
& \frac{d y}{d t}=a_{2} x+b_{2} y+c_{2} x^{2}+d_{2} x y+\mathrm{e}_{2} y^{2}+\ldots
\end{aligned}
$$

Note that the constant terms are missing since $F(0,0)$ and $G(0,0)$ are zero. Near the origin the magnitude of $x$ and $y$ is small and the terms of second and higher order are very small compared to those of first order. Therefore it is reasonable to approximate eqs. (16) and (17) by the linear system:

$$
\begin{aligned}
& \frac{d x}{d t}=a_{1} x+b_{1} y \\
& \frac{d y}{d t}=a_{2} x+b_{2} y
\end{aligned}
$$

where

$$
\begin{aligned}
& a_{1}=\frac{\partial F(\bar{x}, \tilde{y})}{\partial x} \\
& b_{1}=\frac{\partial F(\bar{x}, \tilde{y})}{\partial y}
\end{aligned}
$$

BIOTECHNOLOGY AND BIOENGINEERING, VOL. XII, ISSUE 3 


$$
\begin{aligned}
& a_{2}=\frac{\partial G(\tilde{x}, \tilde{y})}{\partial x} \\
& b_{2}=\frac{\partial G(\tilde{x}, \tilde{y})}{\partial y}
\end{aligned}
$$

It is also reasonable to conjecture that the configuration of the nonlinear system is the same as that of the approximating linear system near the singular point. These expectations can be proven true except for a few borderline cases. . In the borderline cases the linear terms are not sufficient to characterize the solution trajectory patterns. In these cases it is necessary to include second or higher order terms in the analysis.

For the study of singular points of nonliner systems it is seen that the configurations to be expected for the approximating linear system are indispensable. Fortunately, the known theory of linear differential equations is sufficient to classify the configurations which arise. The general solution of the linear approximating equation can be written in the form:

$$
\begin{aligned}
& x=a \mathrm{e}^{\lambda_{1} t}+b \mathrm{e}^{\lambda 2 t} \\
& y=c \mathrm{e}^{\lambda_{1} t}+d \mathrm{e}^{\lambda 2 t}
\end{aligned}
$$

where $a, b, c$, and $d$ are constants and $\lambda_{1}$ and $\lambda_{2}$ are the characteristic roots satisfying the determinant

$$
\left|\begin{array}{ll}
a_{1}-\lambda & b_{1} \\
a_{2} & b_{2}-\lambda
\end{array}\right|=0
$$

The behavior of the solution curves can be related to the values of $\lambda_{1}$ and $\lambda_{2}$.

A singular point is classified as a node if the roots $\lambda_{1}$ and $\lambda_{2}$ of eq. (22) are real and of the same sign. Examples of nodes are shown in Figure 3. If the real roots are both negative the node is stable and the solution trajectories approach the origin as $t$ approaches $+\infty$. This behavior is also apparent from eqs. (20) and (21). If the roots are both positive the node is unstable and the trajectories diverge from the origin as $t$ approaches $+\infty$.

*The rigorous proof of these and other results can be found in any text covering the analysis of nonlinear differential equations, for example, Chapter 7 in Stern. ${ }^{16}$ 


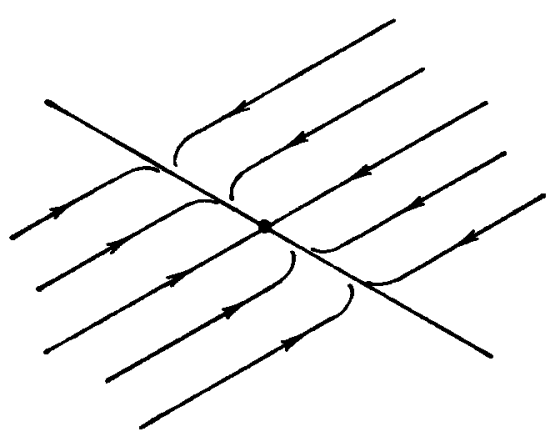

STABLE

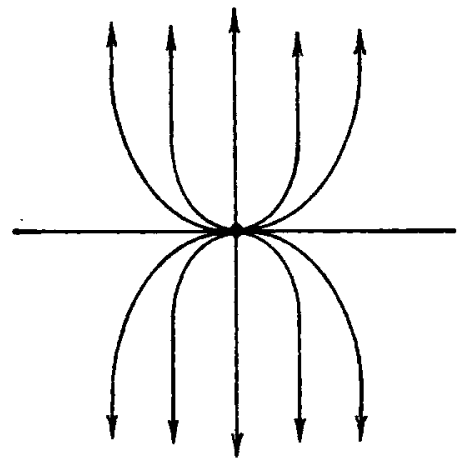

UNSTABLE

Fig. 3. Nodal points.

A singular point is called a saddle point if the characteristic roots are real and of the opposite sign. Equations (20) and (21) show that the motion is unstable. Figure 4 shows a saddle point and its divergent pattern.

A singular point is known as a focal point if the characteristic roots are complex conjugate numbers with nonzero real parts. Near a focal point the solution trajectories behave as spirals as shown in Figure 5. If the real part of the characteristic roots is negative the focal point is stable and the solution trajectories spiral toward the

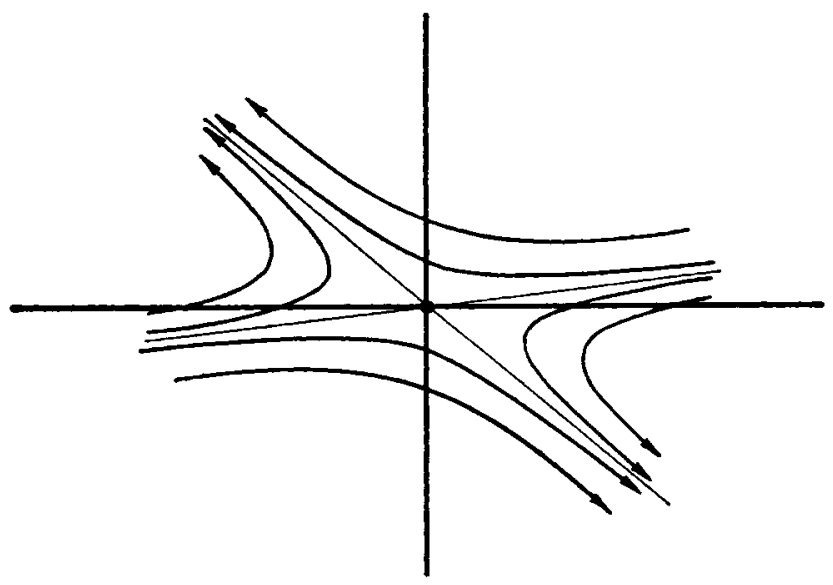

Fig. 4. Unstable saddle point.

BIOTECHNOIOGY AND BIOENGINEERING, VOL. XII, ISSUE 3 


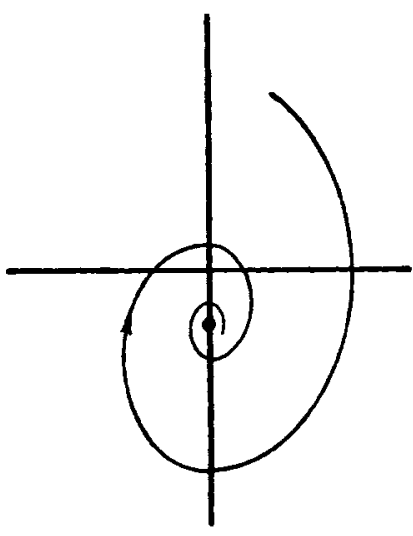

STABLE

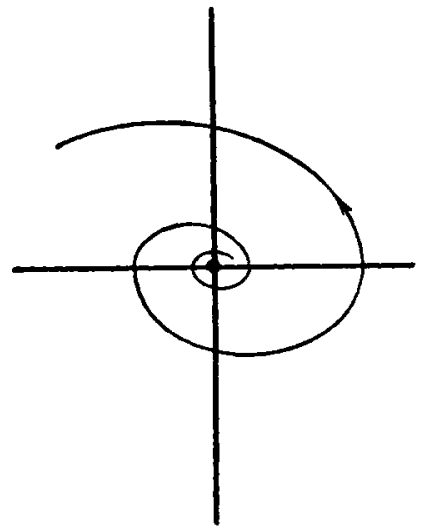

UNSTABLE

Fig. 5. Focal points.

origin as $t$ approaches $+\infty$. If the real part is positive the motion is unstable and the spiral diverges.

If the characteristic roots are pure imaginary numbers all the trajectories in the neighborhood of the singular point are closed curves and correspond to periodic solutions of the linear differential equations. Such a singular point is known as a vortex point and is illustrated in Figure 6 . It is not possible to extrapolate this type of periodic motion from linear approximating equations to the corresponding nonlinear problems. The exact behavior of the nonlinear system will depend on higher order terms.

In summary, second order nonlinear differential equations can be studied in the phase plane by an analysis of the system singular points. In many cases the behavior of the nonlinear system is similar to an approximating linear system near the singular points. It is proposed to use such techniques of analysis to study predatorprey models.

\section{Analysis of Predator-Prey Models}

The three predator-prey models introduced earlier will be studied by the method of phase plane analysis. This analysis will quantitatively demonstrate the differences in the behavior of the solutions of each of the models. The effects of including host nutrients in the model while retaining second order kinetics can be evaluated by comparing the resulting solutions with the Lotka-Volterra model. 


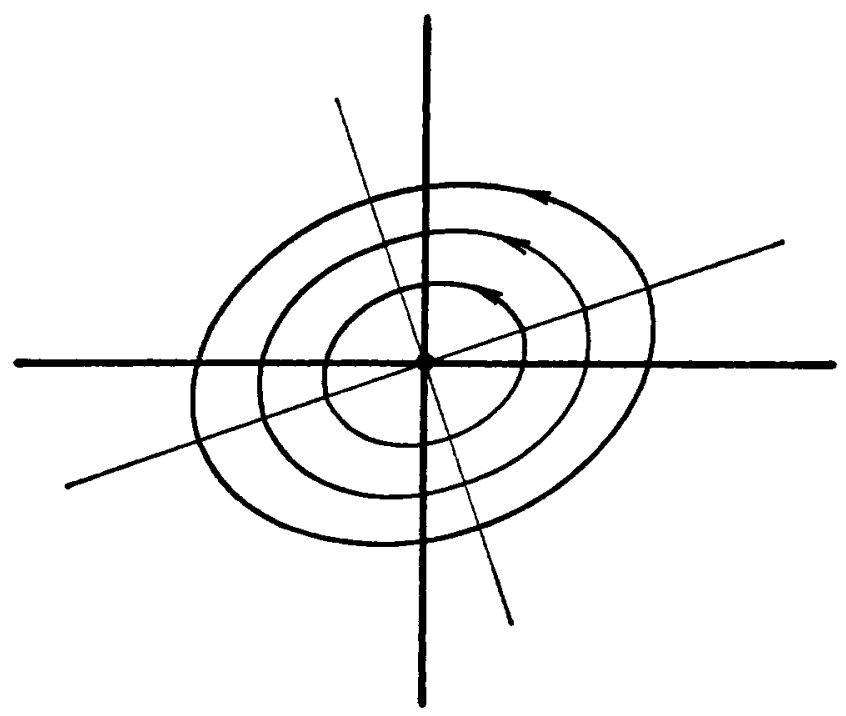

Fig. 6. Vortex point.

The effects of including a saturation in the growth rates of the host and predator can be studied by comparing the resulting solutions with the solutions obtained assuming second order growth kinetics. The transformation,

$$
u=H-\tilde{H}
$$

$v=P-\tilde{P}$ converts eqs. (3) and (4) for the continous culture form of the Lotka-Volterra model to eqs. (23) and (24).

$$
\begin{gathered}
\frac{d u}{d t}=\left(\mu-D-k_{1} \tilde{P}\right) u-\left(k_{1} \tilde{H}\right) v-k_{1} u v \\
-\frac{d v}{d t}=k_{2} \tilde{P} u+\left(k_{2} \tilde{H}-D\right) v+k_{2} u v
\end{gathered}
$$

where $\tilde{H}$ and $\tilde{P}$ are the singular points of eqs. (3) and (4). The linear approximation of eqs. (23) and (24) is

$$
\left[\begin{array}{l}
\frac{d u}{d t} \\
\frac{d v}{d t}
\end{array}\right]=\left[\begin{array}{lr}
\mu-D-k_{1} \tilde{P} & -k_{1} \tilde{H} \\
k_{2} \tilde{P} & k_{2} \tilde{H}-D
\end{array}\right]\left[\begin{array}{l}
u \\
v
\end{array}\right]
$$

BIOTECHNOLOGY AND BIOENGINEERING, VOL. XII, ISSUE 3 
The singular points of the system are obtained by setting the left hand side of eqs. (3) and (4) equal to zero and solving for the appropriate values of $H$ and $P$. The system has two singular points,

$$
\begin{aligned}
& \text { (i) } \quad \tilde{H}=0, \quad \tilde{P}=0 \\
& \text { (ii) } \quad \tilde{H}=\frac{D}{k_{2}}, \quad \tilde{P}=\frac{\mu-D}{k_{1}}
\end{aligned}
$$

where $\mu$ must be greater than $D$ for positive $\tilde{P}$.

Near the singular point $(i)$ the linear approximating equations are

$$
\left[\begin{array}{l}
\frac{d u}{d t} \\
\frac{d v}{d t}
\end{array}\right]=\left[\begin{array}{rr}
\mu-D & 0 \\
0 & -D
\end{array}\right]\left[\begin{array}{l}
u \\
v
\end{array}\right]
$$

and the characteristic roots satisfying

$$
\left|\begin{array}{lr}
\mu-D-\lambda & 0 \\
0 & -D-\lambda
\end{array}\right|=0
$$

are $\lambda_{1}=\mu-D$ and $\lambda_{2}=-D$. The roots are real and of opposite sign for $\mu$ greater than $D$. Singular point $(i)$ is therefore a saddle point.

Near the singular point (ii) the linear approximating equations are

$$
\left[\begin{array}{c}
\frac{d u}{d t} \\
\frac{d v}{d t}
\end{array}\right]=\left[\begin{array}{lr}
0 & -\frac{k_{1}}{k_{2}} D \\
\frac{k_{2}}{k_{1}}(\mu-D) & 0
\end{array}\right]\left[\begin{array}{l}
u \\
v
\end{array}\right]
$$

and the characteristic roots satisfying

$$
\left|\begin{array}{ll}
-\lambda & \frac{k_{1}}{k_{2}} D \\
\frac{k_{2}}{k_{1}}(\mu-D) & -\lambda
\end{array}\right|=0
$$

are $\lambda= \pm j \sqrt{D(\mu-D)}$ for $\mu$ greater than $D$, where $j=\sqrt{-1}$. 


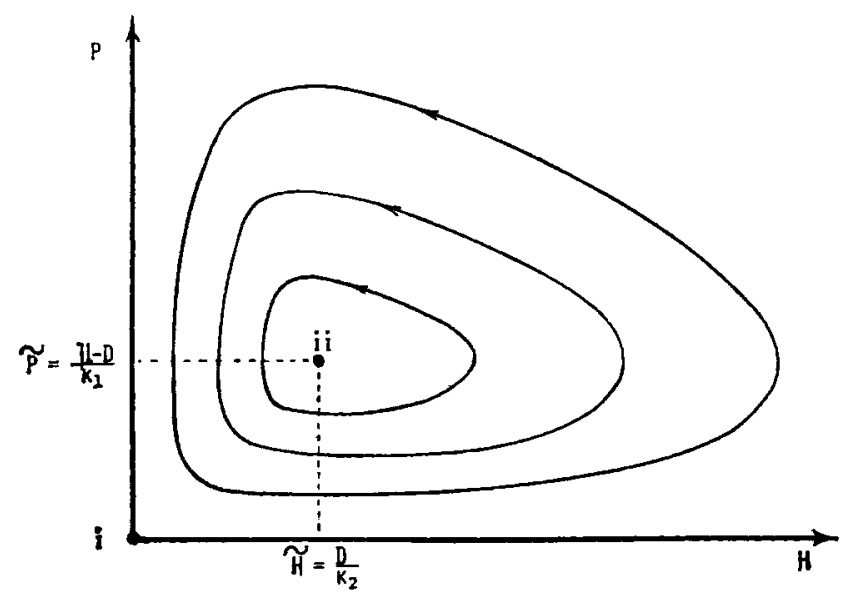

Fig. 7. Solution of Lotka-Volterra model.

Since both roots are imaginary, singular point (ii) is a vortex point.* Figure 7 shows a sketch of the solution trajectories.

The predator-prey model containing second order effects of host food supply given by eqs. (7), (8), and (9) can be written in the dimensionless form

$$
\begin{gathered}
\frac{d x}{d \theta}=1-x-M x y \\
\frac{d y}{d \theta}=M x y-y-N y z \\
\frac{d z}{d \theta}=N y z-z
\end{gathered}
$$

where $\theta=D t, x=s / s_{I}, M=\hat{\mu} s_{I} / D \hat{s}, y=H / Y s_{I}, N=\hat{\eta} Y s_{I} / D \hat{H}$, and $z=P / W Y s_{I}$.

In general, given an initial condition, the solution of eqs. (26), (27), and (28) will trace a curve in the $x y z$-space. However, it can be

*This example, as a borderline case, should include an analysis of second order terms. However, it has been shown by Volterra ${ }^{2}$ that eqs. (3) and (4) possess periodic solutions as indicated by the singular point analysis.

BIOTECHNOLOGY AND BIOENGINEERING, VOL. XII, ISSUE 3 
shown that for any given initial condition the sum $(x+y+z)$ always approaches unity. Let $\phi=x+y+z$, and add eqs. (26), (27) and (28) to obtain:

$$
\frac{d \phi}{d \theta}=1-\phi
$$

the solution of which is $\phi=1-\left(1-\phi_{0}\right) \mathrm{e}^{-\theta}$, where $\phi_{0}$ is the initial value of the sum $\phi$. All the solution trajectories in three dimensional space approach the plane $x+y+z=1$ as $\theta$ approaches infinity. Further, if $\phi_{0}=x+y+z=1$ to start with, then $d \phi / d \theta$ equals zero, and the solution trajectories remain on the plane $x+y+z=1$. Thus the characteristics of the singular points of the three-variable problem represented by eqs. (26), (27), and (28) can be studied as a two dimensional problem on the plane $x+y+z=1$ with the equations

$$
\begin{gathered}
\frac{d y}{d \theta}=M(1-y-z) y-y-N y z \\
\frac{d z}{d \theta}=M y z-z
\end{gathered}
$$

where $y$ is the dimensionless host variable and $z$ is the dimensionless predator variable.

With the change of variables $u=y-\tilde{y}$ and $v=z-\tilde{z}$ the linear approximation of eqs. (29) and (30) can be written as

$$
\left[\begin{array}{l}
\frac{d u}{d \theta} \\
\frac{d v}{d \theta}
\end{array}\right]=\left[\begin{array}{rr}
(M-1)-(M+N) \tilde{z}-2 M \tilde{y} & -(M+N) \tilde{y} \\
N \bar{z} & N \tilde{y}-1
\end{array}\right]\left[\begin{array}{l}
u \\
v
\end{array}\right]
$$

where $\tilde{x}, \tilde{y}$, and $\tilde{z}$ are the values of $x, y$, and $z$ at a singular point. The model given by eqs. (29) and (30) has three singular points

$$
\text { (i) } \begin{aligned}
\tilde{z} & =0 \\
\tilde{y} & =0 \\
\tilde{x} & =1
\end{aligned}
$$




$$
\begin{aligned}
\text { (ii) } \quad \tilde{z} & =0 \\
\tilde{x} & =\frac{1}{M} \\
\tilde{y} & =1-\frac{1}{M} \\
\text { (iii) } \quad \tilde{y} & =\frac{1}{N} \\
\tilde{x} & =\frac{N}{M+N} \\
\tilde{z} & =1-\frac{1}{N}-\frac{N}{M+N}
\end{aligned}
$$

where in each case $\tilde{x}+\tilde{y}+\tilde{z}=1$.

The values of $\bar{x}, \tilde{y}$, and $\tilde{z}$ remain positive provided $N>1, M>$ $N /(N-1)$, and $M-1>(M / N)$.

Near singular point $(i)$ the linear approximating equations become

$$
\left[\begin{array}{l}
\frac{d u}{d \theta} \\
\frac{d v}{d \theta}
\end{array}\right]=\left[\begin{array}{rr}
M-1 & 0 \\
0 & -1
\end{array}\right]\left[\begin{array}{l}
u \\
u
\end{array}\right]
$$

which have characteristic roots $\lambda_{1}=M-1$ and $\lambda_{2}=-1$. Since $M$ must be greater than 1 this singular point is a saddle point.

The linear approximating equations near singular point (ii) are

$$
\left[\begin{array}{c}
\frac{d u}{d \theta} \\
\frac{d v}{d \theta}
\end{array}\right]=\left[\begin{array}{cc}
1-M & -(M+N)\left(1-\frac{1}{M}\right) \\
0 & \frac{N}{M}(M-1)-1
\end{array}\right]\left[\begin{array}{l}
u \\
v
\end{array}\right]
$$

which have characteristic roots $\lambda_{1}=1-M$ and $\lambda_{2}=(N / M)(M-$ 1) -1 . Since it has been required that $M-1>(M / N)$, singular point (ii) is also a saddle.

BIOTECHNOLOGY AND BIOENGINEERING, VOL. XII, ISSUE 3 
Near singular point (iii) the linear approximating equations of eqs. (29) and (30) are

$$
\left[\begin{array}{l}
\frac{d u}{d \theta} \\
\frac{d v}{d \theta}
\end{array}\right]=\left[\begin{array}{cr}
-\frac{M}{N} & -\left(\frac{M+N}{N}\right) \\
\frac{M N}{M+N}-1 & 0
\end{array}\right]\left[\begin{array}{l}
u \\
v
\end{array}\right]
$$

which has the characteristic roots

$$
\lambda=-\frac{1}{2} \frac{M}{N} \pm \frac{1}{2} \sqrt{\left(\frac{M}{N}\right)^{2}-4\left(M-1-\frac{M}{N}\right)}
$$

In this case singular point (iii) is a stable node if $4[M-1-(M / N)]$ $<(M / N)^{2}$ and a stable focal point if $4[M-1-(M / N)]>(M / N)^{2}$. Figures 8 and 9 demonstrate the possibilities.

Higher order sophistication can be obtained in predator-prey models by considering saturation in the growth rates of the predator and the host. This model described by eqs. (12), (13), and (14) can be put in the dimensionless form

$$
\begin{gathered}
\frac{d x}{d \theta}=1-x-\frac{A x y}{a+x} \\
\frac{d y}{d \theta}=\frac{A x y}{a+x}-y-\frac{B y z}{b+y} \\
\frac{d z}{d \theta}=\frac{B y z}{b+y}-z
\end{gathered}
$$

where $\theta=D t, x=s / s_{I}, A=\mu_{\max } / D, a=K / s_{I}, y=H / Y s_{I}, B=$ $\eta_{\max } / D, b=L / Y s_{I}$, and $z=P / Y W s_{I}$. Equations (32), (33), and (34) can be added and integrated to show that the sum $\phi=x+y+$ $z$ approaches 1 as $\theta$ approaches infinity, and that if $\phi_{0}=1$ at $t=0$ then $\phi=1$ for all $t$. Thus the above model can be studied on the plane $x+y+z=1$ with the equations

$$
\begin{gathered}
\frac{d y}{d \theta}=\frac{A(1-y-z) y}{a+1-y-z}-y-\frac{B y z}{b+y} \\
\frac{d z}{d \theta}=\frac{B y z}{b+y}-z
\end{gathered}
$$




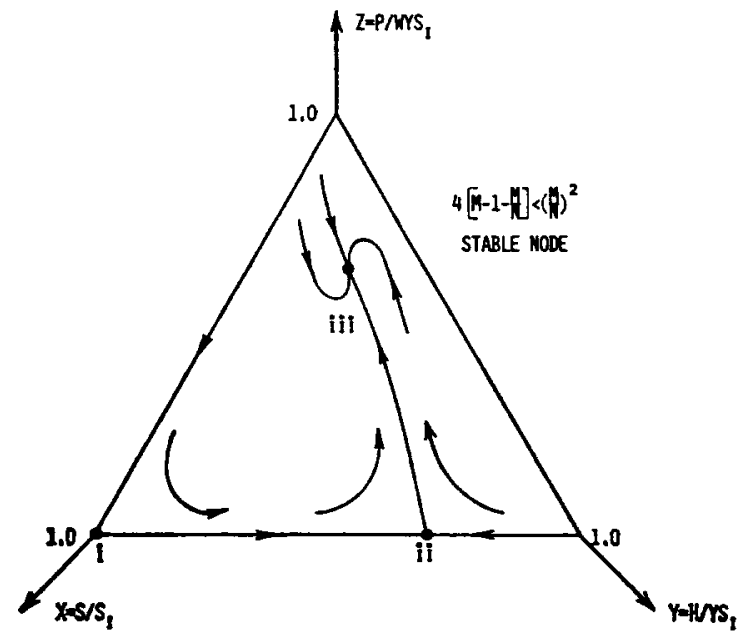

Fig. 8. Phase plane trajectories for predator-prey model without growth rate saturation.

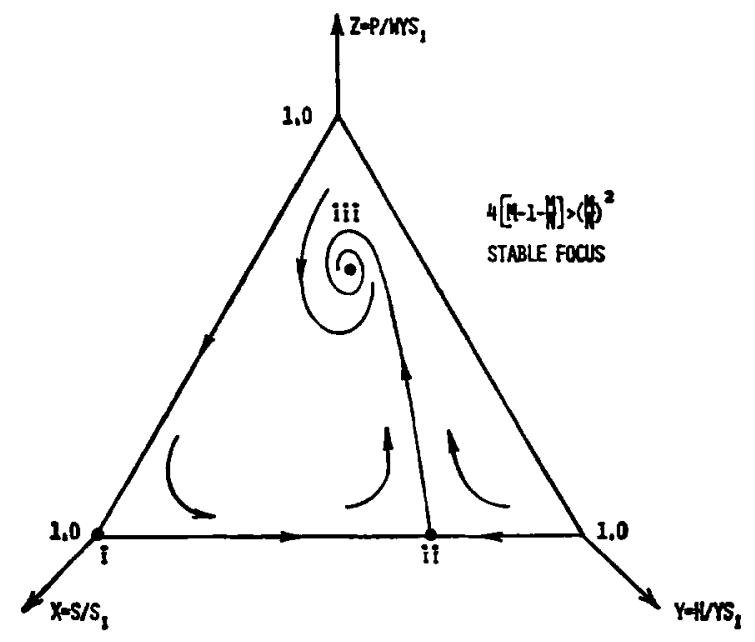

Fig. 9. Phase plane trajectories for predator-prey model without growth rate saturation.

BIOTECHNOLOGY AND BIOENGINEERING, VOL. XII, ISSUE 3 
where $y$ is the dimensionless host variable $H / Y s_{I}$, and $z$ is the dimensionless predator variable $P / Y W s_{I}$.

The change of variables $u=y-\tilde{y}$ and $v=z-\bar{z}$ is introduced to obtain the linear approximation of the model near the singular point $(\tilde{x}, \tilde{y}, \tilde{z})$ which gives

$$
\begin{array}{r}
{\left[\begin{array}{c}
\frac{d u}{d \theta} \\
\frac{d v}{d \theta}
\end{array}\right]=\left[\begin{array}{r}
\frac{A \tilde{y} \tilde{x}}{(a+x)^{2}}-\frac{A \tilde{y}}{a+\tilde{x}}+\frac{A \tilde{x}}{a+\tilde{x}}-1+\frac{B \tilde{y} \tilde{x}}{(b+\tilde{y})^{2}}-\frac{B \tilde{z}}{b+\tilde{y}} \\
\frac{B \tilde{z}}{b+\tilde{y}}-\frac{B \tilde{y} \tilde{z}}{(b+\tilde{y})^{2}} \\
\quad \times \frac{A \tilde{x} \tilde{y}}{(a+\tilde{x})^{2}}-\frac{A \tilde{y}}{a+\tilde{x}}-\frac{B \tilde{y}}{b+\tilde{y}} \\
\frac{B \tilde{y}}{b+\tilde{y}}-1
\end{array}\right]\left[\begin{array}{l}
u \\
v
\end{array}\right]}
\end{array}
$$

The model with saturation of growth rates as given by eqs. (32), (33), and (34) has three singular points

$$
\text { (i) } \begin{aligned}
\tilde{z} & =0 \\
\tilde{y} & =0 \\
\tilde{x} & =1 \\
\tilde{z} & =0 \\
\tilde{x} & =\frac{a}{A-1} \\
\tilde{y} & =1-\frac{a}{A-1}
\end{aligned}
$$

(iii) $\tilde{y}=\frac{b}{B-1}$

$$
\begin{array}{r}
\bar{x}=-\frac{1}{2}\left(a+\frac{A b}{B-1}-1\right)+\frac{1}{2} \sqrt{\left(a+\frac{A b}{B-1}-1\right)^{2}+4 a} \\
\tilde{z}=1-\frac{b}{B-1}+\frac{1}{2}\left(a+\frac{A b}{B-1}-1\right) \\
-\frac{1}{2} \sqrt{\left(a+\frac{A b}{B-1}-1\right)^{2}+4 a}
\end{array}
$$


where each singular point is on the plane $x+y+z=1$. The values of $\bar{x}, \tilde{y}$, and $z$ at each singularity should not be negative. This is guaranteed if $A>a+1, B>b+1$, and $[(B-1) / b][(A-1$ $-a) /(A-1)]>1$.

The linear approximating equation near singular point $(i)$ are

$$
\left[\begin{array}{c}
\frac{d u}{d \theta} \\
\frac{d v}{d \theta}
\end{array}\right]=\left[\begin{array}{lr}
\frac{A}{a+1}-1 & 0 \\
0 & -1
\end{array}\right]\left[\begin{array}{l}
u \\
v
\end{array}\right]
$$

which have characteristic $\operatorname{roots} \lambda_{1}=[A /(a+1)]-1$ and $\lambda_{2}=-1$. Since it has been required that $A>a+1$ singular point $(i)$ is a saddle point.

The model near singular point (ii) has a linear approximation which may be written as

$$
\begin{aligned}
& {\left[\begin{array}{l}
\frac{d u}{d \theta} \\
\frac{d v}{d \theta}
\end{array}\right]=\left[\begin{array}{c}
\frac{-(A-1-a)(A-1)}{a A} \\
0
\end{array}\right.}
\end{aligned}
$$

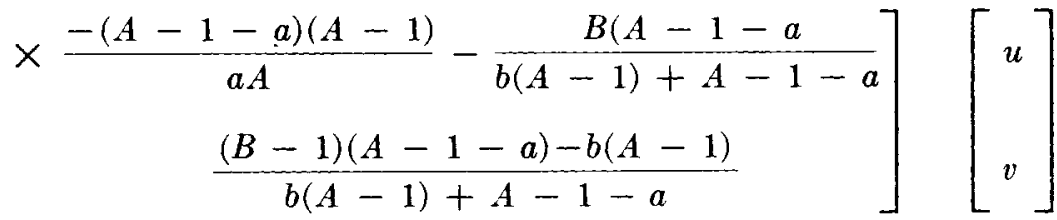

with the characteristic roots

$$
\begin{aligned}
& \lambda_{1}=\frac{-(A-1-a)(A-1)}{a A} \\
& \lambda_{2}=\frac{\frac{B-1}{b} \frac{A-1-a}{A-1}-1}{1+\frac{A-1-a}{b(A-1)}}
\end{aligned}
$$

This singularity represents a saddle point since it has been required that $A>a+1, B>b+1$, and $[(B-1) / b][(A-1-a) /(A-$ 1)] $>1$. 
Near the singular point (iii) the model has a linear approximation given by

$$
\left[\begin{array}{l}
\frac{d u}{d \theta} \\
\frac{d v}{d \theta}
\end{array}\right]=\left[\begin{array}{l}
\frac{1-\tilde{x}-A \tilde{y}+A \tilde{x}}{a+\tilde{x}}-1+\frac{\tilde{z}(1-B)}{b+\tilde{y}} \\
\frac{\tilde{z}(B-1)}{b+\tilde{y}} \\
\times \frac{1-\tilde{x}-A \tilde{y}}{a+\tilde{x}}-1 \\
0
\end{array}\right]\left[\begin{array}{l}
u \\
v
\end{array}\right]
$$

where

$$
\begin{aligned}
\tilde{y} & =\frac{b}{B-1} \\
\bar{x} & =-\frac{1}{2}\left(a+\frac{A b}{B-1}-1\right)+\frac{1}{2} \sqrt{\left(a+\frac{A b}{B-1}-1\right)^{2}+4 a} \\
\bar{z} & =1-\frac{b}{B-1}+\frac{1}{2}\left(a+\frac{A b}{B-1}-1\right) \\
& -\frac{1}{2} \sqrt{\left(a+\frac{A b}{B-1}-1\right)^{2}+4 a}
\end{aligned}
$$

The linear equations have the characteristic roots

$$
\begin{aligned}
& \lambda=\frac{1}{2}\left[\frac{1-\tilde{x}-A \tilde{y}+A \tilde{x}}{a+\tilde{x}}-1+\frac{\tilde{z}(1-B)}{b+\tilde{y}}\right] \\
& \pm \frac{1}{2}\left[\left(\frac{1-\tilde{x}-A \tilde{y}+A \tilde{x}}{a+\tilde{x}}-1+\frac{\tilde{z}(1-B)}{b+\tilde{y}}\right)^{2}\right. \\
&\left.+4\left(\frac{1-\bar{x}-A \tilde{y}}{a+\bar{x}}-1\right)\left(\frac{\tilde{z}(B-1)}{b+\tilde{y}}\right)\right]^{1 / 2}
\end{aligned}
$$

It is possible to show that the above characteristic values can be real and negative indicating that the singularity at (iii) is a stable node. Figure 10 shows the solution trajectory configuration for the case where $A=30, a=.02, b=.1$, and $B=2$. By selecting 


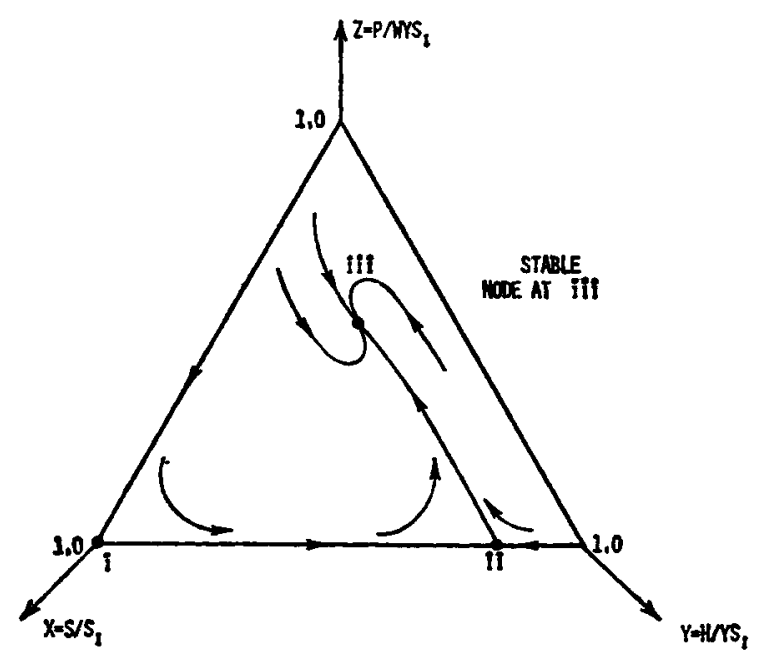

Fig. 10. Phase plane trajectories for predator-prey model with growth rate saturation.

$A=30, a=.02, b=.1$, and $B=3$, the characteristic values become complex conjugates with negative real parts indicating a stable focal point. Figure 11 shows the results for this case. The characteristics roots become complex conjugates with positive real parts when $A=30, a=.02, b=.1$ and $B=4$. In this case singular point (iii) is an unstable focal point. The solution trajectories eventually approach a closed curve on the plane $x+y+z=1$. Figure 12 shows a sketch for this case. The closed curve is called a limit cycle and represents a periodic solution to the predator-prey model. The shape, amplitude, and frequency of the periodic solution are independent of the initial values of $x, y$, and $z$ and depend only on the values of $A, B, a$, and $b$. An approximate solution for this limit cycle has been obtained by Canale $^{10}$ using the method of perturbation.

\section{DISCUSSION AND SUMMARY}

Phase plane sketches of predator-prey relationships can be used to gain a more complete understanding of the Lotka-Volterra model. 


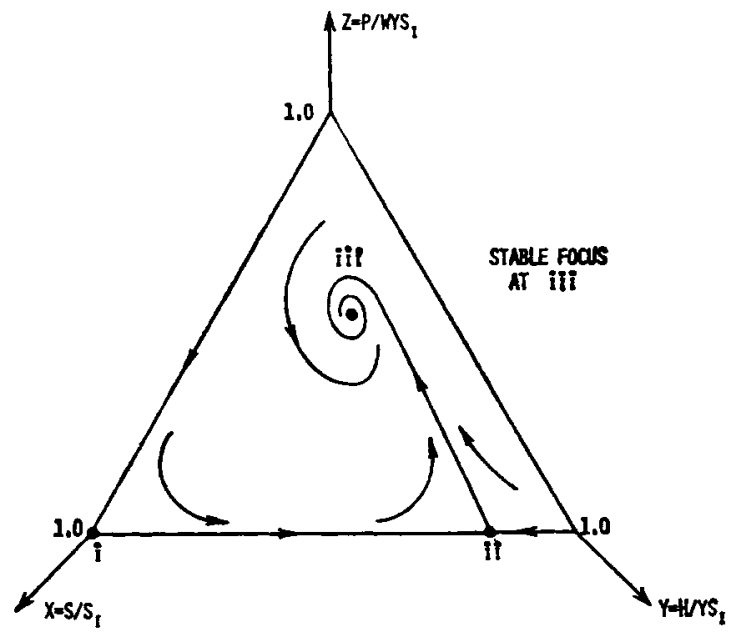

Fig. 11. Phase plane trajectories for predator-prey model with growth rate saturation.

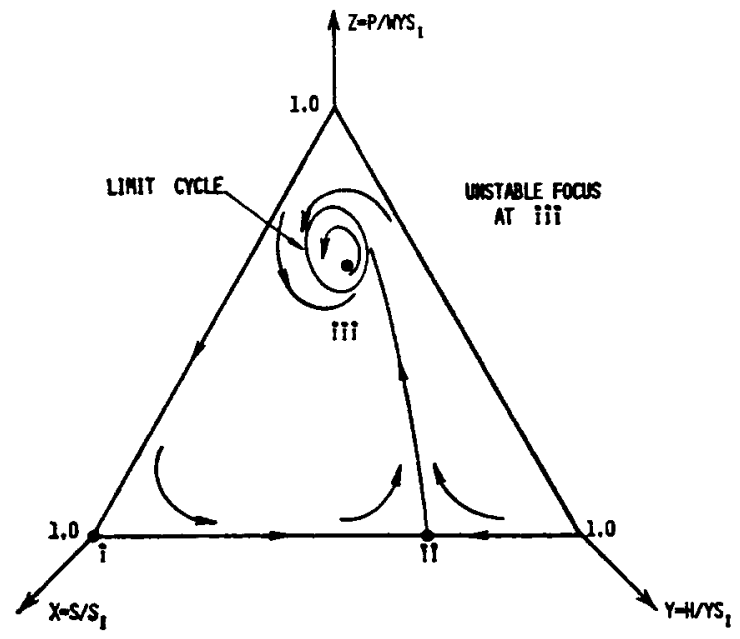

Fig. 12. Phase plane trajectories for predator-prey model with growth rate saturation. 
The effects of two proposed refinements in the model can also be evaluated. Such phase plane sketches can be obtained with the aid of an analysis of the singular points.

The Lotka-Volterra model has only two singular or equilibrium values,

$$
\begin{aligned}
& \text { (i) } \widehat{H}=0, \quad \widetilde{P}=0 \\
& \text { (ii) } \tilde{H}=\frac{D}{k_{2}}, \quad \tilde{P}=\frac{\mu-D}{k_{1}}
\end{aligned}
$$

If $\mu>D$, singular point $(i)$ is a saddle point and singular point (ii) is a vortex point. A sketch of the system is shown in Figure 7 . The solution of this model gives these type oscillations and no other possibilities for solutions exist. Thus in his text Gause ${ }^{12}$ stated: "We expected at the beginning of this chapter to find 'classical' oscillations in numbers arising in consequence of the continuous interaction between predators and prey as was assumed by Lotka and Volterra."* The inflexible nature of this model has brought about these and many other difficulties during recent years. For example, Burbanck and Eisen ${ }^{15}$ have suggested that Gause's experiments failed to produce cycles because of nutritional deficiencies in the system.

The model considering second order effects in the host growth due to a limiting nutrient in the substrate has three equilibrium values:

$$
\text { (i) } \begin{aligned}
\frac{\tilde{P}}{W Y s_{I}} & =0 \\
\frac{\tilde{H}}{Y s_{I}} & =0 \\
\frac{s}{s_{I}} & =1 \\
\text { (ii) } \frac{\tilde{P}}{W Y s_{I}} & =0 \\
\frac{\tilde{s}}{s_{I}} & =\frac{D \hat{s}}{\hat{\mu} s_{I}} \\
\frac{\widetilde{H}}{Y s_{I}} & =1-\frac{D s}{\hat{\mu} s_{I}}
\end{aligned}
$$

*G. F. Gause, "The Stuggle for Existence," The Williams and Wilkins Co., Baltimore, Md., p. 140.

BIOTECHNOLOGY AND BIOENGINEERING, VOL. XII, ISSUE 3 


$$
\text { (iii) } \begin{aligned}
\frac{\tilde{H}}{Y s_{I}} & =\frac{D \hat{H}}{\hat{\eta} Y s_{I}} \\
\frac{s_{I}}{s_{I}} & =\frac{\frac{\hat{\eta} Y s_{I}}{D \hat{H}}}{\frac{\hat{\mu} s_{I}}{D \tilde{s}}+\frac{\hat{\eta} Y s_{I}}{D \hat{H}}} \\
\frac{\tilde{P}}{W Y s_{I}} & =1-\frac{\hat{D} \hat{H}}{\hat{\eta} Y s_{I}}-\frac{\frac{\hat{\eta} Y s_{I}}{D \hat{H}}}{\frac{\hat{\mu} s_{I}}{D \hat{s}}+\frac{\hat{\eta} Y s_{I}}{D \hat{H}}}
\end{aligned}
$$

If $N>1, M>[N /(N-1)]$, and $M-1>(M / N)$ then singular points $(i)$ and (ii) are saddle points and singular point (iii) is either a stable focal point or a stable nodal point. Note that no periodic solutions are possible and that all oscillations eventually decay to steady values.

A more sophisticated model has also been studied by singular point analysis. The model considers a situation where the host growth rate is limited by some nutrient, and where the predator and prey growth rate values saturate with increasing food supply. This model also has three singular points:

$$
\text { (i) } \begin{aligned}
\frac{\tilde{P}}{W Y s_{I}} & =0 \\
\frac{\tilde{H}}{Y s_{I}} & =0 \\
\frac{\widetilde{s}}{s_{I}} & =1 \\
\text { (ii) } \frac{\tilde{P}}{W Y s_{I}} & =0 \\
\frac{\tilde{s}}{s_{I}} & =\frac{K / s_{I}}{\frac{\mu_{\max }}{D}-1} \\
\frac{\tilde{H}}{Y s_{I}} & =1-\frac{K / s_{I}}{\frac{\mu_{\max }}{D}-1}
\end{aligned}
$$




$$
\text { (iii) } \begin{aligned}
\frac{\tilde{H}}{Y s_{I}} & =\frac{L / Y s_{I}}{\frac{\eta_{\max }}{D}-1} \\
\frac{\tilde{s}}{s_{I}} & =-\frac{1}{2}\left[\frac{K}{s_{I}}+\frac{\mu_{\max } L}{D Y s_{I}\left(\frac{\eta_{\max }}{D}-1\right)}-1\right] \\
& +\sqrt{\left[\frac{K}{s_{I}}+\frac{\mu_{\max } L}{D Y s_{I}\left(\frac{\eta_{\max }}{D}-1\right)}-1\right]^{2}+\frac{4 K}{s_{I}}} \\
\frac{\tilde{P}}{W Y s_{I}} & =1-\frac{\tilde{H}}{Y s_{I}}-\frac{\tilde{s}}{s_{I}}
\end{aligned}
$$

If $A>a+1, B>b+1$, and $[(B-1) / b][(\mathrm{A}-1-a) /(A-1)]$ $>1$ singular points $(i)$ and $(i i)$ are saddle points, and singular point (iii) may be a stable node, stable focus, or unstable focus depending on the values of $A, a, B$, and $b$. If singular point (iii) is a stable node no oscillatory phenomena are observed. If the singular point is a stable focal point a damped cycle is produced. This type of behavior is also possible in the model without growth rate saturation. If the singular point becomes an unstable focal point, a limit cycle is produced in the phase plane.

A limit cycle is an oscillation which does not depend on the system initial conditions. This type of behavior has been suggested for predator-prey systems by Gause ${ }^{12}$ from a study of his experimental results: "It must be remarked that the equation (Lotka-Volterra model) does not hold absolutely true because the oscillations (experimental) do not apparently belong to the 'conservative' type. In other terms they do not keep the magnitude initially given them but tend to inherent magnitude of their own."*

Slobodkin ${ }^{13}$ has discussed in great detail the dependence of ecological steady states on outside energy sources. The suggested modifications of the Lotka-Volterra model also provide for a continuous and constant input of energy. The energy is supplied in the form of

*G. F. Gause, "The Struggle for Existance," The Williams and Wilkins Co., Baltimore, Md., p. 137.

BIOTECHNOLOGY AND BIOENGINEERING, VOL. XII, ISSUE 3 
host nutrients and is represented mathematically as $D s_{I}$ in eqs. (7) and (12). In certain cases the explicit inclusion of energy input terms in predator-prey models will lead to limit cycle oscillations. Other cases lead to damped oscillations, or exponential approaches to steady state. By including such energy sources and growth rate saturation effects in the description of predator-prey interactions, a flexible model is obtained which may be useful for simulating experimental data, and may provide a basis for gaining more insight into the basic mechanisms underlying predation.

\section{References}

1. A. J. Lotka, "Elements of Physical Biology," The Williams and Wilkins Co., Baltimore, Md. (1925).

2. V. Volterra, "Variazioni e fluttuazioni del numers d'individui in specie animali conviventi," Mem. Acad. Lineii Roma, 2, 31 (1926).

3. H. R. Bungay, and M. L. Bungay, "Microbial Interactions in Continuous Culture," Advances in Applied Microbiology, 10, 269-290 (1968).

4. J. Monod, "Recherches sur la crossance des cultures bacteriennes," Hermann \& Cie. (1942).

5. D. Herbert, R. Elsworth and R. C. Telling, "The Continuous Culture of Bacteria; a Theoretical and Experimental Study," J. gen. Microbiol, 14, 601-622 (1956).

6. K. L. Schultze, Water and Sewage Works, 111, no. 12, 526, 1964.

7. G. Proper and J. C. Garver, "Mass Culture of the Protozoa Coploda steinii," Biotechnology and Bioengineering, 8, pp. 287-296 (1966).

8. C. R. Curds and A. Cockburn, "Studies on the Growth and Feeding of Tetrahymena pyriformis in Axenic and Monoxenic Culture," Journal of General Microbiology, 54, 343-358 (1968).

9. J. F. Drake, J. L. Jost, A. G. Fredrickson and H. M. Tuschiya, "The Food Chain", Bioregenerative Systems, A conference held in Washington, D.C., November 15-16, 1966 sponsored by NASA and the American Institute of Biological Sciences, NASA SP-165, Library of Congress number 68-60345, U.S. Government Printing Office, Washington, D.C. (1966).

10. R. P. Canale, "Predator-Prey Relationships in a Continuously Fed Biological Reactor," Ph.D. Thesis, Syracuse University, Syracuse, N.Y. (1968).

11. R. P. Canale, "Predator-Prey Relationships in a Model for the Activated Sludge process," Biotechnology and Bioengineering, 11, 887-907, 1969.

12. G. F. Gause, "The Struggle for Existence," Williams \& Wilkins Co., Baltimore, Md. (1934).

13. L. B. Slobodkin, "Growth and Regulation of Animal Populations," Holt, Rinehart and Winston, New York (1961).

14. G. W. Salt, "Predation in an Experimental Protozoan Population (Woodruffin-Paramecium)," Ecological Monographs, 37, No. 2, pp. 113-114 (1967). 
15. W. D. Burbanck and J. D. Eisen, "The Inadequacy of Monobacterially-fed Paramecium aurelia as Food for Didinium nasutum," Journal of Protozoology, 7, 201-206 (1960).

16. T. E. Stern, "Theory of Nonlinear Networks and Systems," AddisonWesley, Reading, Mass, (1965).

Received September 19, 1969

Revised February 6, 1970 